\title{
Study of Key Non-dimensional Parameters for Wave Drag Reduction with High-Frequency Repetitive Laser Pulse Energy Depositions
}

\author{
Zexu Qing ${ }^{1,2}$, Yanji Hong ${ }^{1}$, Diankai Wang ${ }^{1}$ and Bin Zhang ${ }^{2}$ \\ ${ }^{1}$ State Key Laboratory of Laser Propulsion \& Application, Equipment Academy, 101416 Beijing, China \\ ${ }^{2}$ School of Aeronautics and Astronautics, Shanghai Jiao Tong University, 200240 Shanghai, China
}

\begin{abstract}
The problem of wave drag reduction with high-frequency repetitive laser pulse energy depositions is multivariable. Three key non-dimensional parameters, non-dimensional energy, non-dimensional depositing position and Mach number, were constructed from a number of original variables by using Buckingham pi theorem. Influences of these non-dimensional parameters on energy deposition performance, namely drag reduction and energy deposition efficiency, were investigated numerically by solving three-dimensional Navier-Stokes equations with an upwind scheme. Optimizing method of non-dimensional energy and non-dimensional depositing position is proposed. Drag reduction and energy deposition efficiency have exponential relationships with non-dimensional energy; Drag reduction and energy deposition efficiency have quadratic relationships with non-dimensional depositing position. Drag reduction has exponential relationship with freestream Mach number and energy deposition efficiency has quadratic relationship with Mach number. Non-dimensional laser energy and non-dimensional depositing position should be optimized synthetically for a given freestream.
\end{abstract}

\section{Introduction}

At the present state of art, realizing practicable hypersonic flight is hampered by great drag wave, which even accounts for more than half of total drag in some cases. High-frequency repetitive laser pulse energy deposition is a promising technique to reduce supersonic wave drag with the merit of good controllability, high energy density and energy efficiency. The disturbed region (core) with lower density and higher temperature, generated by the laser-induced optical breakdown, interacts with the bow shock. A mitiagation of the bow shock accompanied by drag and heat flux reduction can be observed.

Experimental study of a single laser pulse energy deposition was performed over a sphere in a supersonic flow with Mach number of 3.45 by Adelgren et al. [1]. Erdem et al. [2] mitigated the bow shock by single-pulse energy and high voltage discharge. Study conducted by Kim et al. [3] shown that a drag reduction about $21 \%$ corresponding to energy deposition efficiency of $\sim 10$ could be obtained at Mach number of 1.92 utilizing the laser whose maximum frequency and power was up to $50 \mathrm{kHz}$ and $400 \mathrm{~W}$ respectively. Numerical results indicated the existence of optimal depositing position [4].

P. K. Tret'yakov realized steady high-frequency repetitive laser pulse energy depositions for wave drag reduction for the first time [5]. It was shown that drag reduction increased with increasing of laser frequency in the premise that laser average power kept nearly unchanged. A series of experiments were conducted in a Mach-1.92, in-draft wind tunnel by A. Sasoh's group. It was shown that energy deposition efficiency monotonically decreased with increasing laser average power and drag reduction was proportional to laser average power [6]. Riggins et al. [7] investigated the drag reduction utilizing a full Navier-Stokes CFD code. According to their study, drag force was reduced to value as low as $30 \%$ associated with energy deposition efficiency of up to 33 at Mach numbers of 6.5 and 10 without optimization. Wave drag reduction by a single pulse energy deposition was calculated in sphere model by S. Taguchi et al. [8] and Y. Ogino [9]. The drag reduction was proportional to the freestream Mach number under the constant energy deposition condition in Taguchi's study. Existence of optimal depositing position was also proved in Taguchi's work. Ogino determined that the reduced energy was proportional to the square of the freestream Mach number. It is seen that the energy deposition efficiency increases with increasing of Mach number from V. A. Levin's work [10]. Nearly linear (but very shallow) decreasing trend in the percentage of drag reduced with increasing Mach number (lower than 6) was shown in T. Taylor's study [11].

Parameters for wave drag reduction with highfrequency repetitive laser pulse energy depositions are so numerous, including laser energy magnitude, energy depositing position, Mach number, pressure and temperature of freestream, size and shape of the body etc., that it is necessary to construct key non-dimensional 
parameters to reduce the difficulty and cost of experiments. Based on these key non-dimensional parameters, a series of numerical experiments were carried out and more general laws about the effects of these parameters on energy deposition performance were obtained.

\section{Dimensional analysis}

There are two important criterions in evaluating energy deposition performance, namely the drag reduction $\eta$ and energy deposition efficiency $S$, defined as:

$$
\begin{aligned}
& \eta=\frac{\Delta D}{D_{\text {ref }}}=\frac{D_{\text {ref }}-D_{\text {mod }}}{D_{\text {ref }}} \\
& S=\frac{\left(D_{\text {ref }}-D_{\text {mod }}\right) \cdot V_{\infty}}{P}=\frac{D_{\text {ref }} \cdot V_{\infty}}{P} \cdot \eta
\end{aligned}
$$

where $\Delta D, D_{\text {ref }}$ and $D_{\text {mod }}$ are the drag modification, baseline drag without laser energy deposition and modified drag, respectively; $P$ is the laser power and $V_{\infty}$ is the freestream velocity.

In the premise that optical breakdown took place, we expect $\eta$ and $S$ to depend on blunt body diameter $D$, freestream velocity $V_{\infty}$, freestream density $\rho_{\infty}$, freestream temperature $T_{\infty}$, freestream specific heat at constant pressure $c_{p}$, laser power $P$, distance from body nose to breakdown $L$, kinetic viscosity $\mu$. Without any a priori knowledge about the variation of $\eta$ and $S$, we can use common sense to write

$$
\begin{aligned}
& \eta=f\left(D, V_{\infty}, \rho_{\infty}, T_{\infty}, P, c_{p}, L, \mu\right) \\
& S=g\left(D, V_{\infty}, \rho_{\infty}, T_{\infty}, P, c_{p}, L, \mu\right)
\end{aligned}
$$

Due to four fundamental dimensions and eight physical variables, there are four dimensionless $\pi$ products. Choose $D, V_{\infty}, \rho_{\infty}$ and $T_{\infty}$ as the arbitrarily selected sets of four physical variables. These $\pi$ products are

$$
\begin{array}{ll}
\pi_{1}=\frac{P}{D^{\alpha_{1}} V_{\infty}^{\beta_{1}} \rho_{\infty}^{\gamma_{1}} T_{\infty}^{\kappa_{1}}} & \pi_{2}=\frac{L}{D^{\alpha_{2}} V_{\infty}^{\beta_{2}} \rho_{\infty}^{{ }^{\gamma_{2}}} T_{\infty}^{{ }^{\kappa_{2}}}} \\
\pi_{3}=\frac{c_{p}}{D^{\alpha_{3}} V_{\infty}^{\beta_{3}} \rho_{\infty}^{\gamma_{3}} T_{\infty}^{\kappa_{3}}} & \pi_{4}=\frac{\mu}{D^{\alpha_{4}} V_{\infty}^{\beta_{4}} \rho_{\infty}^{\gamma_{4}} T_{\infty}^{{ }^{\kappa_{4}}}}
\end{array}
$$

The results of dimensional analysis is

$$
\eta=f\left(\frac{P}{D^{2} V_{\infty}^{3} \rho_{\infty}}, \quad \frac{L}{D}, \quad c_{p} \frac{T_{\infty}}{V_{\infty}^{2}}, \quad \frac{\mu}{D V_{\infty} \rho_{\infty}}\right)
$$

Non-dimensional energy $\varepsilon$ is defined as the ratio of laser power $P$ and freestream enthalpy flux $\dot{H}$

$$
\varepsilon=\frac{P}{\dot{H}}=\frac{P}{\rho_{\infty} c_{p} T_{\infty} V_{\infty} D^{2}}
$$

Introducing Mach number and Reynolds number, we have

$$
\begin{aligned}
& \eta=f\left(\varepsilon, \frac{L}{D}, \quad M a, \quad R e\right) \\
& S=f\left(\varepsilon, \frac{L}{D}, \quad M a, \quad R e\right)
\end{aligned}
$$

In Equation (6), $\eta$ and $S$ are expressed as general functions of non-dimensional energy $\varepsilon$, non-dimensional depositing position $L / D$, freestream Mach number and Reynolds number $R e$. Due to the little influence of viscosity on wave drag, $R e$ is ignored and three key nondimensional parameters are obtained.

\section{Study of key non-dimensional parameters}

\subsection{Computational methodology}

Three-dimensional compressible Navier-Stokes equations were solved with an upwind scheme to simulate the flow. The calculation adopted second-order accurate scheme based on the finite volume method and the domain decomposition of the structural grid. An implicit difference method was employed. An upwind scheme based on a rotating Riemann solver was used to calculate the inviscid flux. A perfect gas model was used. Laser energy was deposited instantaneously with frequency of $150 \mathrm{kHz}$ because frequency has little influence on energy deposition performance when frequency is high enough. Evolution of the plasma was ignored. Laser energy was deposited in the area with a diameter of $1 \mathrm{~mm}$. twodimensional blunt body model with width of $20 \mathrm{~mm}$ was adopted. Mach number of freestream was 2.5 except for Section 3.4. Static pressure and temperature of the freestream were $58 \mathrm{kPa}$ and $130 \mathrm{~K}$ respectively. The structured grids were generated with commercial software GridgenV15, and the total number was $9 \times 10^{4}$. No-slip wall conditions were applied on the surface of the blunt body. The grid and definition of the boundary were shown in Figure 1. The CFD code was developed on our own.

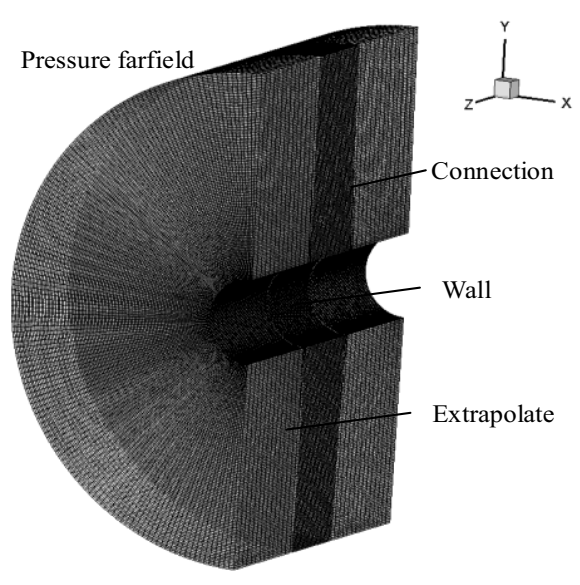

Figure 1. Grid and boundary definition.

\subsection{Non-dimensional energy $\varepsilon$}


To study the influence of non-dimensional energy on performance of laser energy deposition, non-dimensional depositing position and Mach number are fixed to 1 and 2.5 respectively. Flow fields with different $\varepsilon$ were shown in Figure 2. The hot core becomes bigger and density becomes lower when more laser energy is deposited, bringing more drag reduction. However, bigger area of the hot core means that the influence is moving away from stagnation point, which results in the decrease of $S$.

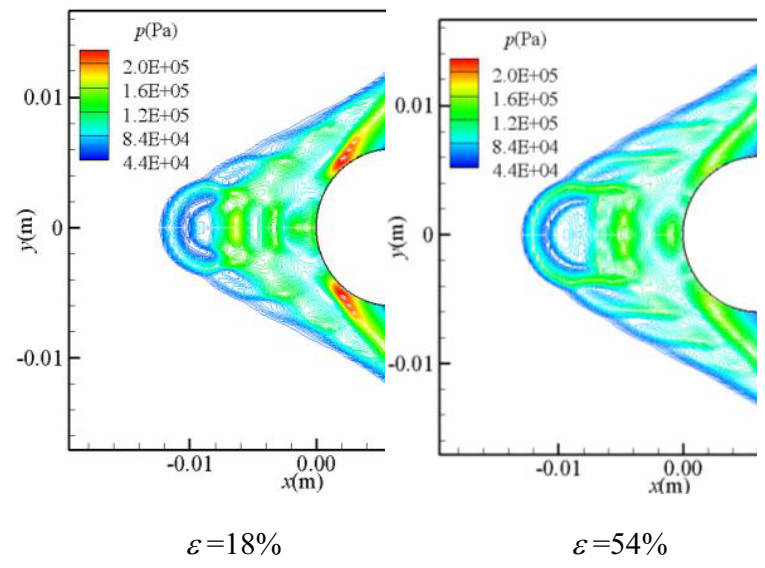

Figure 2. Flow fields with $\varepsilon=18 \%$ and $\varepsilon=54 \%$.

As shown in Figure 3, three blunt bodies with different diameters go through similar performance trend with the increase of non-dimensional energy and the energy deposition performance is finely discriminating which demonstrates the reliability of key nondimensional parameters. With increasing of $\varepsilon, \eta$ increases and $S$ decreases.
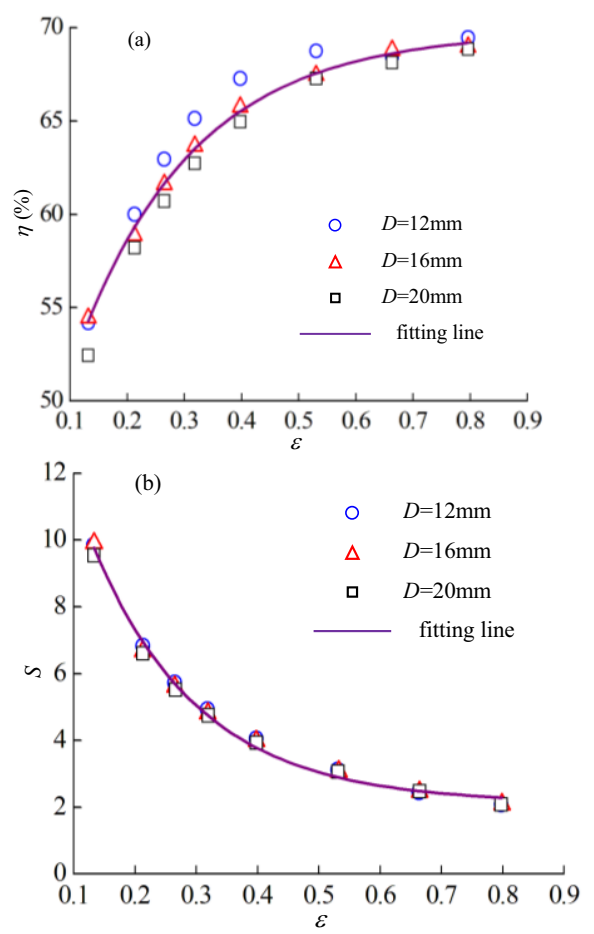

Figure 3. Influence of non-dimensional energy on performance of laser energy deposition at $L / D=1, M a=2.5$ : (a) drag reduction; (b) energy deposition efficiency.
Fitting data points of Figure 3 with exponential curves, we have

$$
\eta=-29.49 \mathrm{e}^{\frac{-\varepsilon}{0.21}}+69.80 \quad S=16.52 \mathrm{e}^{\frac{-\varepsilon}{0.17}}+2.13
$$

From Equation (7), it is found that $\eta$ seems to converge to $69.8 \%$ and $S$ converges to 2.13 as $\varepsilon$ goes to infinity for the given calculation condition. $\eta$ and $S$ can be determined approximately by Equation (7) if $\varepsilon$ locates in the range of $0.1 \sim 0.8$. Keeping Equation (6) in mind, it's reasonable to infer that constants in Equation (7) have relations to $L / D$ and $M a$. While more numerical experiments are needed to reveal those relationships.

\subsection{Non-dimensional depositing position $L / D$}

Influence of $L / D$ on performance of laser energy deposition is shown in Figure 4. The diameter of blunt body is chosen to be $10 \mathrm{~mm}$. Freestream condition is the same as what mentioned above. Large or small $L / D$ makes the compression wave front caused by interaction of blast wave and bow shock too close to the body surface, so energy deposition performance is not good enough, as shown in Figure 5. Cases with different $\varepsilon$ is also included, symbolized by different colours.

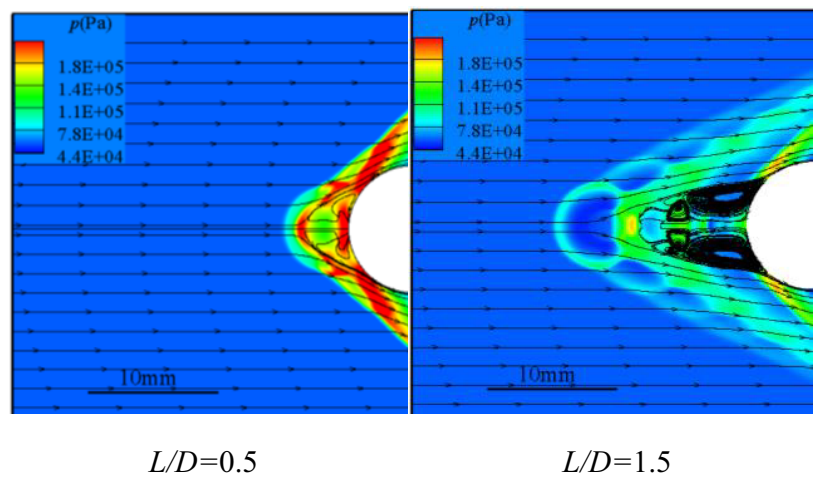

Figure 4. Flow fields with different $L / D, \varepsilon=0.066$.

Data points in Figure 5 were fitted with quadratic curves. The expressions have the form of

$$
\eta, S=A(L / D)^{2}+B(L / D)+C
$$

So $(-B / 2 A)$ represents the optimal $L / D$ with the highest $\eta$ and $S$. A positive correlation of $(-B / 2 A)$ with $\varepsilon$ can be found in Figure 6 .

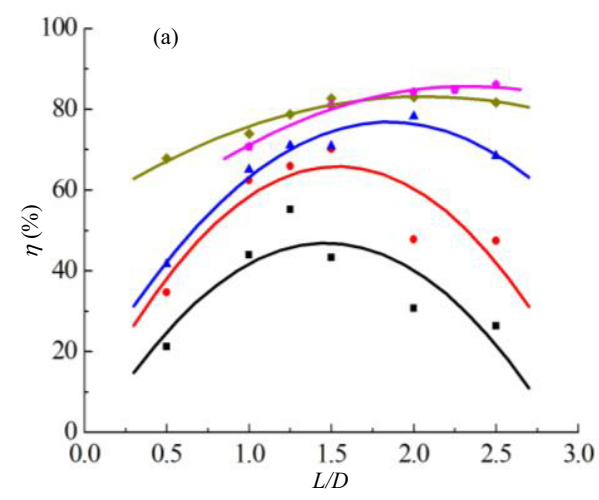




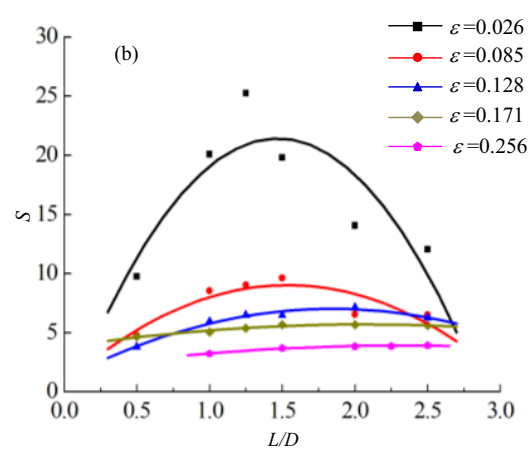

Figure 5. Influence of non-dimensional depositing position on performance of laser energy deposition at $P=1000 \mathrm{~W}, D=10 \mathrm{~mm}$, $M a=2.5$ : (a) drag reduction; (b) energy deposition efficiency.

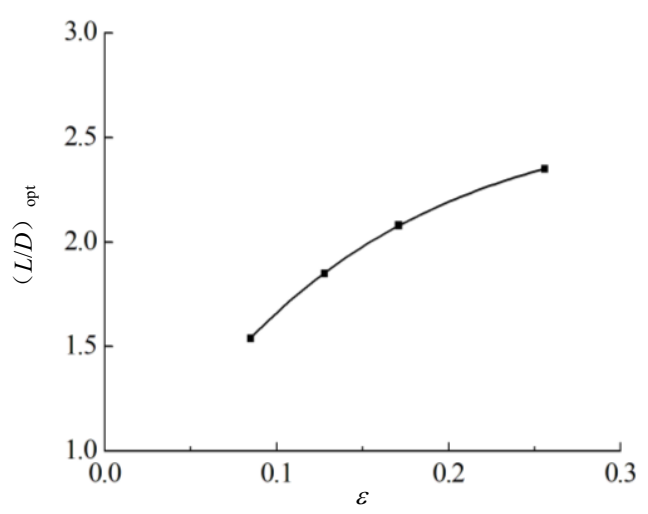

Figure 6. Relationship of non-dimensional energy with optimal depositing position.

\subsection{Mach number Ma}

Fixing $\quad P=1000 \mathrm{~W}, \quad D=12 \mathrm{~mm}, \quad L / D=1, \quad$ numerical experiments with diffenent $M a$ are conducted. Figure 7 shows two typical flow fields. As shown in Figure 8, for the constant laser power $P$, it is commonsensible that $\eta$ decreases with the increase of $M a$, which can be described roughly by exponential equation, meaning that more laser energy is needed to obtain enough drag reduction in high Mach number situation.
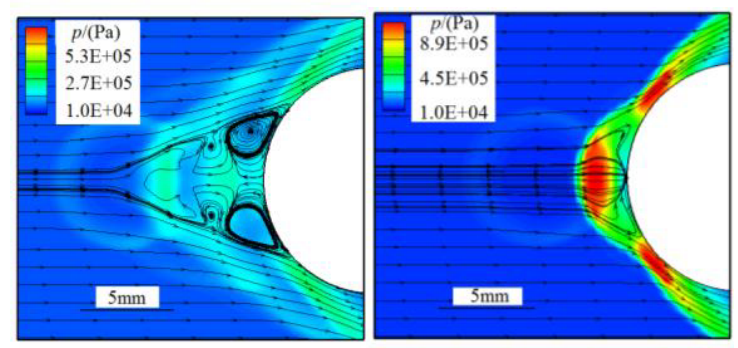

Figure 7. Pressure contour and streamlines of different $M a$ : left, $M a=3$; right, $M a=5$.

On the other hand, with increasing of $M a, S$ increases firstly and then decreases, like a quadratic relation. $S$ reaches the maximum when $M a$ is $\sim 5.5$. If $M a$ is too large, the generation of baroclinic vortex will be limited due to the decrease of shock standoff distance, resulting the decrease of $\eta$ and $S$. If $M a$ is too small, meaning the low flow speed, the hot core will expand too large before interacting with bow shock, resulting in the decrease of $S$.

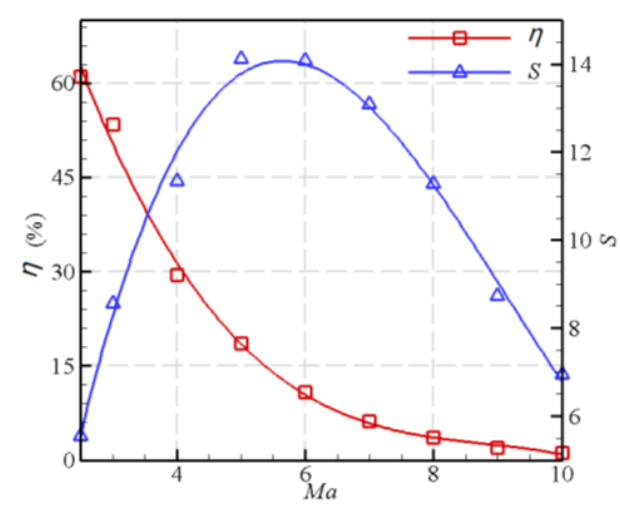

Figure 8. Influence of freestream $M a$ on performance of laser energy deposition

\section{Summary}

Buckingham pi theorem is a powerful tool in analyzing numerical experiments of wave drag reduction with highfrequency repetitive laser pulse energy depositions. Dimensional analysis shows that non-dimensional energy $\varepsilon$, non-dimensional depositing position $L / D$ and Mach number $M a$ are the key non-dimensional parameters. $\varepsilon$ has an exponential relation with $\eta$ and $S ; L / D$ has a quadratic relation with $\eta$ and $S$; $M a$ has exponential and quadratic relation with $\eta$ and $S$ respectively. Influence of these three key non-dimensional parameters on laser deposition performance intertwines each other and integrated optimization method is proposed.

\section{References}

1. R. G. Adelgren, H. Yan, G. S. Elliott, D. D. Knight, T. J. Beutner, A. A. Zheltovodov, AIAA J., 43, 2 256-269 (2005)

2. E. Erdem, L. Yang, K. Kontis, AIAA 2011-1027 (2011)

3. J. H. Kim, A. Matsuda, T. Sakai, A. Sasoh, AIAA 2010-5104 (2010)

4. L. N. Myrabo, Yu. P. Raizer, M. N. Shneider, Beamed energy propulsion: Third international symposium on beamed energy propulsion, AIP Publishing, 485-498 (2005)

5. P. K. Tret'yakov, G. N. Grachev, A. I. Ivanchenko, et al., Dokl. Phys., 39, 6 415-416 (1994)

6. A. Sasoh, Y. Sekiya, T. Sakai, J. H. Kim, A. Matsuda. AIAA J., 48, 12 2811-2817 (2010)

7. D. Riggins, H. F. Nelson, E. Johnson., AIAA 981647 (1998)

8. S. Taguchi, N. Ohnishi, M. Furudate, et al, AIAA 2007-1235 (2007)

9. Y. Ogino, N. Ohnishi, S. Taguchi, K. Sawada, Phys. Fluids., 21, 6 (2009)

10. V. A. Levin, N. A. Afonia, V. G. Gromov, AIAA99-4967 (1999)

11. T. Taylor, AIAA 2004-131 (2004) 